\title{
Forschungsberichte
}

\section{Lärmempfindlichkeit und mobiles Musikhören im urbanen Umfeld}

\author{
Noise Sensitivity and Mobile Music Listening in Urban Areas
}

\author{
Jasha Kettel ${ }^{1}$, Mia Kuch¹, Clemens Wöllner ${ }^{1}$
}

[1] Institut für Systematische Musikwissenschaft, Universität Hamburg, Hamburg, Deutschland.

\section{Zusammenfassung}

Während die Weltgesundheitsorganisation schon seit geraumer Zeit auf die negativen Auswirkungen von Lärm hinweist, sind nach wie vor viele Menschen im Wohnumfeld und auch im öffentlichen Raum hohen Lärmpegeln ausgesetzt. Ob Musik im Umgang mit Umweltlärm genutzt wird und welche Zusammenhänge mit der individuellen Lärmempfindlichkeit im urbanen Raum bestehen, sollte im Rahmen der vorliegenden Studie untersucht werden. Dazu wurde ein Online-Fragebogen erstellt, der neben der Häufigkeit des mobilen Musikhörens auch das Motiv des bewussten Lärmausblendens und das Hörverhalten sowie die individuelle Lärmempfindlichkeit erfasste. Die Ergebnisse von 149 Befragten zeigen, dass diejenigen Personen, die empfindlicher auf Lärm reagieren, unterwegs und auch allgemein weniger Musik hören als weniger lärmempfindliche Personen. Wenn lärmempfindliche Personen unterwegs Musik hören, dann jedoch häufiger als andere, um sich von störenden Geräuschen abzuschotten. Kopfhörer mit lärmabschottender Wirkung (Noise-Cancelling, OverEars, In-Ears) wurden von 107 der Befragten verwendet, wobei lärmempfindliche Personen diese Art der Kopfhörer nicht häufiger nutzten. Die Bedeutung der Lärmempfindlichkeit für das Musikhörverhalten im urbanen Raum steht im Zusammenhang mit mehreren Faktoren, die weiterführende Untersuchungen unter Einbeziehung von Pegelmessungen und situativ empfundener Lärmbelastung empfehlen lassen.

Schlüsse/wörter: Lärm, Funktionen des Musikhörens, Hörgewohnheiten, öffentliche Verkehrsmittel, Altagspendeln, auditive Blase

\begin{abstract}
Although the World Health Organization has long emphasized the negative effects of noise, many people still find themselves exposed to high noise levels in their residential environment and in urban areas. The present study examines individual strategies in dealing with noise in relation to music listening and individual noise sensitivity. An online questionnaire was developed that addresses the frequency of mobile music listening, the sensitivity to noise in urban areas and the choice of listening to music for supressing noise. Findings from 149 respondents indicate that those who respond more sensitively to noise choose to listen less often to music generally and on the move. Yet if noise-sensitive individuals do listen to music while commuting, then they do so more often than less-sensitive individuals for the function of blocking out noise. Noise-cancelling headphones or noise-reducing headphones (over-ears, in-ears) were used by 107 of respondents, but noise-sensitive individuals did not appear to prefer using these types of headphones. The importance of noise sensitivity for listening to music in urban areas is related to several factors, and further studies could include direct noise measurements and situationally perceived noise.
\end{abstract}

Keywords: noise, functions of music listening, listening habits, public transport, commuting, auditory bubble

Jahrbuch Musikpsychologie, 2021, Vol. 30: Musikpsychologie - Empirische Forschungen - Ästhetische Experimente, Artikel e99, https://doi.org/10.5964/jbdgm.99

Eingereicht: 2021-02-22. Akzeptiert: 2021-10-13. Publiziert (VoR): 2021-11-09.

Begutachtet von: Jan Hemming; Felix Thiesen.

*Korrespondenzanschrift: Institut für Systematische Musikwissenschaft, Universität Hamburg, Neue Rabenstraße 13, 20354 Hamburg, Deutschland. EMail: mia.kuch@uni-hamburg.de

Dieser Open-Access-Artikel steht unter den Bedingungen einer Creative Commons Namensnennung 4.0 International Lizenz, CC BY 4.0 (https://creativecommons.org/licenses/by/4.0/deed.de). Diese erlaubt für beliebige Zwecke (auch kommerzielle) den Artikel zu verbreiten, in jedwedem Medium zu vervielfältigen, Abwandlungen und Bearbeitungen anzufertigen, unter der Voraussetzung, dass

der Originalartikel angemessen zitiert wird. 
Der Einfluss des Lärms auf die physische und psychische Gesundheit ist ein zentrales Thema unserer Zeit. Schätzungen zufolge leben mehr als die Hälfte der EU-Bürger/-innen in Gebieten, in denen akustische Belastungen durch hohe Lärmpegel nachweisbar sind (World Health Organization [WHO], 1999). Einer aktuellen Studie der Europäischen Umweltbehörde (European Environment Agency [EEA], 2020) zufolge haben bisherige Maßnahmen zur Reduzierung der Geräuschpegel nur unzureichenden Erfolg. Der Schwerpunkt der Untersuchungen, die bislang zur Lärmbelastung existieren, liegt vor allem auf den Auswirkungen im häuslichen Umfeld, wobei sich hohe Lärmbelastungen auch im öffentlichen Raum, zum Beispiel beim Pendeln, feststellen lassen. Individuelle Bewältigungsstrategien im Umgang mit Lärm, wie die mobile Musiknutzung in öffentlichen Verkehrsmitteln unter Berücksichtigung der subjektiven Lärmempfindlichkeit, sind dabei kaum erforscht.

\section{Richtlinien zu Lärm}

Im Allgemeinen wird Lärm von der WHO (1999) wie auch dem Bundesministerium für Umwelt, Naturschutz und nukleare Sicherheit (BMU; BMU, 2014) unabhängig der Örtlichkeit als unerwünschtes, lautes Geräusch definiert. Sind die Ursachen des Lärms menschliche Aktivitäten im öffentlichen Raum, die somit potenziell eine Vielzahl an Personen betreffen (z. B. Straßen-, Schienen-, Flugverkehr), wird von Umweltlärm gesprochen (EEA, 2020). Zur Bestimmung von Geräuschen und Lärm wird in der Regel der genormte und frequenzabhängige Schalldruckpegel $\mathrm{dB}(\mathrm{A})$ verwendet. Die Grenze zwischen Geräuschen, die als störend oder nicht störend empfunden werden, unterliegt jedoch maßgeblich einer subjektiven Bewertung, wobei Lärmbelastungen enorme Auswirkungen wie Schlafstörungen, körperliche und psychologische Störungen, Abnahme der Leistungsfähigkeit und sonstige Belästigungsreaktionen zur Folge haben können (WHO, 1999).

Laut einer Umfrage aus dem Jahr 2018 fühlen sich in Deutschland ca. 75\% der Bevölkerung durch Straßenlärm im Wohnumfeld gestört (Umweltbundesamt [UBA], 2020). Ungefähr 40\% der Bevölkerung im europäischen Raum sind tagsüber von durchschnittlichen Straßenlärm-Pegeln von über $55 \mathrm{~dB}(\mathrm{~A})$ betroffen, bei $20 \%$ der Befragten liegen diese sogar jenseits von 65 dB(A) (WHO, 1999, S. vii). Das entspricht etwa einem normalen Gespräch (55 dB[A]) oder der Lautstärke eines Rasenmähers (65 dB[A]). Durchschnittliche Geräuschpegel am Tag von $60 \mathrm{~dB}(\mathrm{~A})$ erhöhen das Risiko für Herzerkrankungen, ab 65-70 dB(A) sind Auswirkungen auf den Stoffwechsel, Hormonhaushalt, Stresssymptome und Bluthochdruck zu beobachten, ab $75-85 \mathrm{~dB}(\mathrm{~A})$ ist eine dauerhafte Minderung der Hörleistung möglich (BMU, 2017; WHO, 1999, 2018). Dahingegen sind bereits ab $25 \mathrm{~dB}(\mathrm{~A})$ Schlaf- und Konzentrationsstörungen feststellbar (BMU, 2017), wonach durch Lärm bedingte psychologische Beschwerden in der Bevölkerung verbreiteter sein dürften als physische. Da sich eine kleine Bevölkerungsgruppe auch tagsüber bereits von durchschnittlichen Geräuschpegeln unter $50 \mathrm{~dB}(\mathrm{~A})$ moderat und von Geräuschpegeln unter $55 \mathrm{~dB}(\mathrm{~A})$ stark belästigt fühlen, empfiehlt die WHO (1999) tagsüber entsprechend durchschnittliche Schallpegel von max. $50 \mathrm{~dB}(\mathrm{~A})$.

Inwiefern der Anteil der Personen, die sich von Lärm belästigt fühlen, proportional zu einem erhöhten Schallpegel steigt, ist bisher kaum erforscht. In Montreal konnte jedoch gezeigt werden, dass mit zunehmender Distanz zwischen Wohnumfeld und externen Lärmquellen wie Straßen-, Schienen-, oder Fluglärm eine Reduktion des Schallpegels einhergeht und das individuelle Belästigungsempfinden durchschnittlich abnimmt (Ragettli et al., 2015).

Die Empfehlungen der WHO beziehen sich auf dauerhafte Schalldruckpegel im häuslichen Umfeld. Doch nicht nur diese Art der Geräuschbelastung kann negative Auswirkungen haben, auch bei zeitlich begrenzten 
Lärmbelastungen, wie beispielsweise bei der Nutzung öffentlicher Verkehrsmittel, handelt es sich um psychische und physiologische Stressoren (Koslowsky et al., 1995), die zu Erschöpfung, Hörminderung, Nervosität, Kopfschmerzen und Gereiztheit führen können (Koushki et al., 2002).

\section{Lärmpegel im öffentlichen Verkehr}

Zu Geräuschpegeln in öffentlichen Verkehrsmitteln liegen international einzelne Studien vor, die einen Eindruck über die Lärmbelastung von Fahrgästen vermitteln (siehe Tabelle 1). Yao und Kollegen (2017) führten in Toronto Messungen in U-Bahn- und Buslinien durch und stellten hohe Lärmpegel von durchschnittlich 76,8 $\mathrm{dB}(\mathrm{A})$ innerhalb der U-Bahnen und 76,3 dB(A) in Bussen fest. In New York (Neitzel et al., 2009) ergaben weitere Messungen durchschnittliche Schallpegel von 73,3 dB(A) in S-Bahnlinien (commuter rails), 75,3 dB(A) in Buslinien beziehungsweise $79,3 \mathrm{~dB}(\mathrm{~A})$ in U-Bahnlinien. Innerhalb der Stadtlinienbusse in Kuwait konnten Lärmpegel in Höhe von durchschnittlich 79,0 dB(A) festgestellt werden (Koushki et al., 2002) und in der U-Bahn von Madrid (Tabacchi et al., 2011) wurden mit durchschnittlich 78,2 dB(A) vergleichbare Schalldruckpegel gemessen wie in Toronto oder New York.

\section{Tabelle 1}

Mittlere Lärmpegel innerhalb von öffentlichen Verkehrsmitteln in verschiedenen internationalen Städten und außerhalb von öffentlichen Verkehrsmitteln in Deutschland, in $d B(A)$

\begin{tabular}{lccc}
\hline Ort & S-Bahn & U-Bahn & Bus \\
\hline Toronto & - & 76,8 & 76,3 \\
New York & 73,3 & 79,3 & 75,3 \\
Kuwait & - & - & 79,0 \\
Madrid & - & 78,2 & - \\
Deutschland $^{\text {a }}$ & 70 - 80 & - & - \\
\hline a Messung außerhalb der Fahrzeuge in einem Abstand von 7 m & &
\end{tabular}

aMessung außerhalb der Fahrzeuge in einem Abstand von $7 \mathrm{~m}$.

Für das deutschsprachige Schienennetz liegen Informationen des Bundesministeriums für Verkehr und digitale Infrastruktur (BMVI) lediglich für die Lärmpegel außerhalb der Fahrzeuge vor. Der Informationsbroschüre „Lärmschutz im Schienenverkehr“ (BMVI, 2019) ist zu entnehmen, dass S- und Regionalbahnen im Abstand von $7 \mathrm{~m}$ einen Lärmpegel von $70-80 \mathrm{~dB}(\mathrm{~A})$ erzeugen.

Die Ergebnisse der Studien zeigen allesamt eine Überschreitung der Grenzwerte, die den Richtlinien der WHO zufolge als belästigend empfunden werden können (WHO, 1999, 2018). Es handelt sich für die Einzelperson zwar nicht um dauerhafte Belastungen, dennoch dürfte sich ein Großteil der Personen, die öffentliche Verkehrsmittel nutzen, von der Lautstärke beeinträchtigt fühlen. Die vorgestellten Studien empfehlen auf Basis der Messungen verschiedene Maßnahmen zur Reduzierung der Lautstärke im öffentlichen Verkehr, wie beispielsweise durch die Modernisierung der Züge und Infrastruktur sowie regelmäßige Wartungsarbeiten. Auch die Verwendung von Gehörschutz wird als Handlungsoption für die Fahrgäste genannt (Neitzel et al., 2009; Yao et al., 2017).

\section{Lärmempfindlichkeit, Lärmbelastung und Musikhören}

Sowohl die Wahrnehmung von Geräuschen als Lärm als auch die Reaktionen darauf können individuell stark variieren, weswegen für die Evaluierung von Lärm die Berücksichtigung der subjektiven Komponente hinsicht- 
lich des empfundenen Belästigungsgrads von zentraler Bedeutung ist. Physikalische Eigenschaften wie die Lautstärke tragen ebenso dazu bei wie die persönliche Einstellung zu Lärm (Job, 1988). Das subjektive Empfinden der Lärmbelästigung kann über die individuelle Lärmempfindlichkeit (engl. noise sensitivity) eingeschätzt werden. Hierbei handelt es sich um ein Persönlichkeitsmerkmal, das Einstellungen gegenüber Umweltlärm impliziert (Ellermeier et al., 2001).

Zur Messung der Lärmempfindlichkeit existieren einzelne Inventare, beispielsweise der englischsprachige Fragebogen (Weinstein) Noise Sensitivity Scale (WNSS; Weinstein, 1978), der über 21 Items verfügt. Laut eines Überblickartikels über die WNSS wurde festgestellt, dass es sich um den am häufigsten verwendeten Fragebogen in diesem Themenfeld handelt, der in verschiedenen Sprachen und in verschiedenen Kurzversionen verfügbar ist (Worthington, 2018). Für den deutschsprachigen Raum handelt es sich bei dem Fragebogen zur Erfassung der individuellen Lärmempfindlichkeit von Zimmer und Ellermeier (1998) um ein weiteres Inventar. Dieser Fragebogen ist als Gegenentwurf zum WNSS zu verstehen, der laut Zimmer und Ellermeier (1998) zumindest in Teilen auf "Studenten und Akademiker zugeschnitten“ sei (S. 12) und außerdem nur wenige Situationen im Altag abbilde. Sie konzipierten ihren Fragebogen folglich so, dass er auf eine breite Bevölkerungsschicht anwendbar ist, sich auf Geräusche verschiedener Situation bezieht und Bereiche wie „[...] Alltag, Freizeit, Gesundheit, Schlaf, Kommunikation und Arbeit/Leistung“ erfasst (S. 12).

Die Lärmempfindlichkeit scheint mit verschiedenen Faktoren im Zusammenhang zu stehen. Es konnte beispielsweise festgestellt werden, dass die Lärmempfindlichkeit sowohl mit zunehmender Lärmbelastung (Miedema \& Vos, 2003) als auch mit steigendem Alter zunimmt (Miedema \& Vos, 1999; Shepherd et al., 2015). Empirische Ergebnisse zeigen außerdem, dass sich lärmempfindliche Personen stärker von Umweltlärm belästigt fühlen als lärmunempfindliche Personen; dieser Effekt wird mit steigender Lautstärke der Umweltgeräusche verstärkt (Schutte et al., 2007). Insgesamt bewerten lärmempfindliche Personen Geräuschquellen als lauter und unangenehmer und fühlen sich bei identischer Lärmbelastung auch stärker davon belästigt im Vergleich zu lärmunempfindlicheren Personen (Ellermeier et al., 2001; Sung et al., 2017). Die Art der Geräuschquelle ist bei der Bewertung für beide Gruppen von Bedeutung, so werden Zuggeräusche unangenehmer bewertet als andere Geräuschquellen (Ellermeier et al., 2001). Außerdem steigen mit zunehmender Lärmempfindlichkeit der empfundene Stress sowie allgemeine Stressreaktionen (z. B. Miyakawa et al., 2006).

Psychologische Variablen können dabei auf das Zusammenspiel von Lärmbelastung und gesundheitlichen Folgen einwirken. Lercher (1996) verweist in seinem zusammenfassenden Forschungsartikel unter anderem auf die appraisal theory, wonach durch das Erkennen einer Lärmbelastung, einer passenden Bewältigungsstrategie sowie einer Neubewertung der Situation negative Effekte reduziert werden können. Außerdem wurden verschiedene Umgangsweisen mit Lärm festgestellt (coping strategies), beispielsweise Vermeidungstaktiken oder der Versuch, die Kontrolle über vorhandene Stressoren zu gewinnen. Eine aktive Bewältigungsstrategie, um die Kontrolle über das Gehörte zu erhalten und sich von Lärm abzulenken, könnte dabei das Maskieren von akustischen Umweltreizen durch Musik darstellen.

In einer Studie zur Lärmbelastung im Wohnumfeld gaben Kinder beispielsweise an, dass sie bei Lärm Musik abspielen (Haines et al., 2003), und auch im schulischen Kontext konnte festgestellt werden, dass sich Schüler/-innen, die Musik hören, von einer hohen Umweltlärmbelastung weniger stark belästigt fühlen als diejenigen, die keine Musik hören (Lundquist et al., 2000). Ein ähnlicher Effekt ist ebenfalls bei Erwachsenen zu beobach- 
ten. In einem Experiment von Shu und Kollegen (2018) zeigte sich, dass Musik die subjektiv wahrgenommene Lärmbelästigung durch Straßenverkehr oder Baumaßnahmen reduzieren kann.

Obwohl es sich bei dieser Strategie um das Hinzufügen einer zusätzlichen akustischen Schallquelle handelt, unterscheidet sich Musik zweifelsohne auch für mobil Hörende entscheidend von Lärm (vgl. Reybrouck et al., 2019). Während Musik als Bewältigungsstrategie intendiert eingesetzt wird, hinsichtlich Lautstärke und Auswahl eigenständig kontrolliert werden kann und ein intentional strukturiertes Klangbild aufweist, zeichnen sich Lärmquellen durch hohe, potenziell gesundheitsgefährdende Schalldruckpegel sowie ein chaotisches Klangbild aus und sind unerwünscht. Doch auch das Hören von Musik kann gesundheitliche Schäden nach sich ziehen (Airo et al., 1996; Rice et al., 1987; Shimokura \& Soeta, 2012). Insbesondere zur Lärmbewältigung ist es erforderlich, dass die Musik eine bestimmte Lautstärke erreicht, um Umweltgeräusche erfolgreich ausblenden zu können. Dementsprechend nimmt in Abhängigkeit der Lautstärke vorhandener Hintergrundgeräusche auch die Lautstärke der rezipierten Musik zu.

Inwieweit die individuelle Lärmempfindlichkeit im Zusammenhang mit der Musiknutzung steht, ist bislang weitgehend unerforscht. Lediglich Kliuchko und Kollegen (2015) untersuchten diesen Zusammenhang und stellten fest, dass lärmempfindliche Personen insgesamt weniger passiv Musik im Hintergrund hören und Musik insgesamt auch als weniger wichtig in ihrem Alltag bewerten als lärmunempfindliche Personen.

\section{Mobiles Musikhören zur Lärmbewältigung}

Offen bleibt bislang, inwieweit sich diese Erkenntnis auf den öffentlichen Raum übertragen lässt, beispielsweise beim Pendeln, wobei Personen insbesondere im urbanen Umfeld oft einer hohen Lärmbelästigung ausgesetzt sind. In einer Interviewstudie stellte Skånland (2011) fest, dass während des Pendelns häufig Stress empfunden wird, der sich vor allem auf überfüllte Verkehrsmittel und Lärm zurückführen lässt. Neben den emittierten Fahrzeuggeräuschen selbst fühlen sich die befragten Personen auch stark von den Unterhaltungen anderer Fahrgäste gestört. Musik scheint in diesen Situationen dabei helfen zu können, Umweltgeräusche auszublenden, wodurch sich die Befragten besser auf sich selbst konzentrieren und letztlich ihre wahrgenommene Lebensqualität sowie ihr Wohlbefinden während des Pendelns verbessern können.

Musikhören während des Pendelns wird unter dem Begriff des mobilen Musikhörens untersucht, das als ortsunabhängige Höraktivität in der Öffentlichkeit definiert und durch die Nutzung mobiler Endgeräte und Kopfhörer ermöglicht wird (Kuch \& Wöllner, 2021). Bereits mit dem Aufkommen des Walkmans in den 1980er Jahren wurde die mobile Musiknutzung als Akt der Selbstermächtigung im urbanen Raum verstanden (Hosokawa, 1984) und spätestens mit den empirischen Untersuchungen von Bull (2000) zeigte sich, dass damit bis zu einem gewissen Grad Kontrolle über die eigene Wahrnehmung zurückgewonnen werden kann. Dieses Gefühl von Autonomie findet in einer sogenannten „auditory bubble“ (Bull, 2005, S. 344) statt, was auf eine vorrangig auditive Kontrolle hindeutet. Das Zurückziehen in diese Blase eröffnet wiederum verschiedene Strategien, die von mobilen Hörer/-innen angewendet werden können, um ihre individuellen Erfahrungen zu beeinflussen und ihr Verhältnis zur Umwelt aktiv gestalten zu können. Dies gelingt beispielsweise durch den Einfluss auf das Zeitempfinden (Wöllner \& Hammerschmidt, 2021) oder die aktuelle Stimmung und Gefühlslage (Randall \& Rickard, 2017), die auch im mobilen Kontext von hoher Relevanz sind (Heye \& Lamont, 2010; Kuch \& Wöllner, 2021; Simun, 2009; Skånland, 2013). Darüber hinaus nutzen mobile Musikhörer/-innen Musik, um sich mental leichter von der Umwelt entkoppeln und Umweltgeräusche ausblenden zu können: „Users might be aiming to 
block out any external sound that they might otherwise hear in the street or elsewhere" (Bull, 2000, S. 186). Die akustische Kontrolle durch die Musiknutzung dient demnach neben anderen Funktionen dazu, Umweltärm auszublenden und dessen negative Auswirkungen zu reduzieren.

Weitere Hinweise dazu finden sich in einzelnen Aussagen von Bulls Interviewstudie aus dem Jahr 2005, in der einige Befragte die Stadt als etwas Unangenehmes, Lautes und Chaotisches beschreiben, worauf sie gereizt reagieren. Diesen Umstand können sie durch die Musiknutzung aber abmildern, wie die Befragte Joey im Interview veranschaulicht (Bull, 2005):

The iPod drowns out all this noise and chaos ... The subway is noisy with scratchy announcements and squeaking wheels. The noise makes me irritated and nauseous, but if I have my headphones on it blocks the noises and makes me less irritable and impatient. If I wait on the subway platform for a half-hour I do not mind if I have my iPod to listen to. (S. 353)

Auch Simun (2009) und Chen (1998) stellten in qualitativen Untersuchungen fest, dass Personen, die unterwegs Musik hören, sich leichter von der Umgebung distanzieren können und damit auch von einer hohen Lärmbelastung ablenken können. Heye und Lamont (2010) konnten die Relevanz dieser Nutzungsstrategie in ihrem Fragebogen quantitativ belegen, wodurch die Bedeutung des Nutzungsmotivs des mobilen Musikhörens, störende Umweltgeräusche auszublenden, zusätzlich hervorgehoben wird.

Neben der Musik selbst spielt auch die Kopfhörerwahl eine Rolle, ob Umweltgeräusche wahrgenommen werden oder nicht. Ohrumschließende Kopfhörer (Over-Ears) und Kopfhörer, die in den Gehörgang eingeführt werden (In-Ears) gewährleisten eine abschottende Wirkung von Umweltgeräuschen, während ohraufliegenden Kopfhörer (On-Ears) oder Ohrhörer (Earbuds), die üblicherweise beim Kauf von Smartphones mitgeliefert werden und dadurch weit verbreitet sind, diesbezüglich einen geringeren Effekt aufweisen. Darüber hinaus gibt es sogenannte Noise-Cancelling-Kopfhörer (NC-Kopfhörer), die in Echtzeit Interferenzen erzeugen und Außengeräusche auslöschen, wodurch eine optimale Abschottung gewährleistet wird und die Musik auch bei niedrigerer Lautstärke gut wahrgenommen werden kann.

\section{Zielsetzung}

Die vorliegende Arbeit untersucht quantitativ Zusammenhänge zwischen der individuellen Lärmempfindlichkeit, der Nutzung verschiedener Fortbewegungsarten, der mobilen Musiknutzung sowie dem Musik-Hörverhalten. Es soll geklärt werden, ob sich die Lärmempfindlichkeit von Personen auf die Häufigkeit auswirkt, unterwegs Musik zu hören und ob das Nutzungsmotiv, störende Umweltgeräusche auszublenden, für lärmempfindliche Personen von größerer Bedeutung ist als für weniger lärmempfindliche Personen. Außerdem soll untersucht werden, ob sich die Häufigkeit der mobilen Musiknutzung in Abhängigkeit von der Verkehrsmittelwahl unterscheidet und auch einen Effekt auf die subjektiv empfundene Lautheit der Musik hat.

Dazu wurde ein Online-Fragebogen entwickelt, der die subjektive Lärmempfindlichkeit und Daten zum Musik-Hörverhalten erfasst. Basierend auf der bisherigen Forschung wurden folgende Hypothesen entwickelt:

H1: Die Lärmempfindlichkeit von Personen wirkt sich auf die Häufigkeit aus, mit der unterwegs Musik gehört wird.

H2: Personen mit einer erhöhten Lärmempfindlichkeit geben häufiger an, Musik zum Ausblenden störender Geräusche zu hören, als Personen mit einer niedrigeren Lärmempfindlichkeit. 
H3: Die Nutzung öffentlicher Verkehrsmittel verändert das Hörverhalten, indem öfter mobil Musik gehört wird sowie der subjektiven Einschätzung nach auch lauter Musik gehört wird.

Außerdem soll untersucht werden, ob die individuelle Lärmempfindlichkeit mit weiteren Variablen wie dem Alter, der Kopfhörerwahl, der subjektiv empfundenen Lautheit der unterwegs gehörten Musik oder der Einwohnerzahl (Unterscheidung von ländlichen und urbanen Gebieten im Sinne der Lärmbelastung) im Zusammenhang steht.

\section{Methode}

\section{Teilnehmer/-innen der Online-Befragung}

Verbreitet wurde der Link zur Umfrage über verschiedene Social-Media-Plattformen, über Fachschaftsräte mehrerer deutscher Universitäten, Aushänge und in Lehrveranstaltungen. Daraus resultierte eine Stichprobe von 149 Personen, die zwischen 18 und 65 Jahren alt waren, das mittlere Alter lag bei 29,68 Jahren (SD = 9,03). Der Datensatz umfasste mit 54,4\% etwas mehr Frauen als Männer (divers oder keine Angabe: je $n=1$ ), $75,5 \%$ der Befragten verbrachten ihren Alltag überwiegend in einer Großstadt (> 500.000 Einwohner/-innen). Die Teilnehmenden hatten die Möglichkeit, an der Verlosung eines Amazon-Gutscheins teilzunehmen.

\section{Fragebogen}

Die Daten wurden mit Hilfe eines Online-Fragebogens über Limesurvey erhoben, welcher sich aus fünf Teilen zusammensetzte (siehe Anhang). Im ersten Teil wurde das mobile Musikhörverhalten erfragt (Häufigkeit der mobilen Musiknutzung, Art der Kopfhörer, Musiklautstärke), die Häufigkeit der allgemeinen Musiknutzung sowie die Häufigkeit der Nutzung verschiedener Fortbewegungsarten (S-Bahn/U-Bahn, Bus, zu Fuß, Fahrrad, Motor$\mathrm{rad} /$ Roller, Auto). Im zweiten Teil des Fragebogens wurde die Zustimmung zum Nutzungsmotiv erhoben, unterwegs Musik zu hören, um störende Geräusche auszublenden. Obwohl verschiedene Funktionen im Kontext des mobilen Musikhörens bekannt sind (siehe z. B. Bull, 2000), weist nur dieses Nutzungsmotiv einen direkten Bezug zur mobilen Musiknutzung im Umgang mit Lärm auf, weswegen wir uns mit diesem Forschungsanliegen auf dieses beschränkten. Im dritten Teil wurden Fragen zur Lärmempfindlichkeit gestellt, wofür ein gekürzter Fragebogen entwickelt wurde, der sowohl eine Auswahl an Items von Zimmer und Ellermeier (1998) als auch von Worthington (2018; Weinstein, 1978) umfasst, der für den mobilen Kontext besonders relevant ist und gleichzeitig den Bearbeitungsumfang möglichst gering hält. Dabei wurden lediglich die Items verwendet, die sich nicht ausschließlich auf das Wohnumfeld der Befragten beziehen, um möglichst genaue Informationen über die subjektive Wahrnehmung der Lärmbelastung im öffentlichen Raum zu erhalten. Darüber hinaus wurden drei Items entwickelt, die Bezug auf die Erkenntnisse der mobilen Musiknutzung nehmen (z. B. Stressempfinden, Unterhaltungen anderer).

Negativ gepolte Items zur Messung der Lärmempfindlichkeit wurden in der Datenauswertung umkodiert, um aus der Gesamtzahl der einzelnen Fragen einen Summenwert bilden zu können, der die individuelle Lärmempfindlichkeit abbildet. Die 12 Items der Lärmempfindlichkeit weisen eine hohe interne Konsistenz auf ( $\alpha$ $=$,82). Die Skala zur Messung der Häufigkeit der (mobilen) Musiknutzung umfasste sieben Ausprägungen. Das Nutzungsmotiv des mobilen Musikhörens und die individuelle Lärmempfindlichkeit wurden anhand einer 5- beziehungsweise 6-stufigen Likert-Skala gemessen, die Häufigkeit der Fortbewegungsart anhand einer 4-stufigen Likert-Skala. Anschließend erfolgte die Abfrage der demographischen Daten (siehe Anhang für den 
1. - 4. Teil des Fragebogens). Darüber hinaus wurde den Befragten optional die Möglichkeit gegeben, ihr Musikhörverhalten frei zu beschreiben, dieser fünfte Teil wird an dieser Stelle nicht analysiert.

\section{Ergebnisse}

\section{Mobiles Musikhören und Lärmempfindlichkeit}

In der Stichprobe gaben 136 Personen an, unterwegs Musik zu hören $(91,3 \%)$ und sind demnach als „mobile Musikhörer/-innen“ zu bezeichnen, während 13 Personen unterwegs nie Musik hören. Unter den mobilen Hörer/-innen zeigten sich deutliche individuelle Unterschiede hinsichtlich der Häufigkeit der mobilen Musiknutzung: $17,7 \%$ hören mehrmals im Monat unterwegs Musik, 23,5\% mehrmals in der Woche, 24,3\% täglich bis zu einer Stunde, 25,7\% täglich zwischen 1 und 2 Stunden und 6,6\% hören täglich zwischen 2 und 4 Stunden unterwegs Musik. Keine Person gab an, täglich mehr als vier Stunden unterwegs Musik zu hören. Drei Personen gaben an $(2,2 \%)$, die Häufigkeit der mobilen Musiknutzung nicht einschätzen zu können, diese wurden fallweise aus den Analysen ausgeschlossen.

Aus den 12 Items zur Messung der individuellen Lärmempfindlichkeit wurden personenbezogene Summenwerte berechnet, die zwischen 26 und 69 lagen $(M=47,45, S D=8,69)$. Die Lärmempfindlichkeit wies gemäß Shapiro-Wilk-Test eine Normalverteilung auf, $p>, 05$. Die Befragten wurden basierend auf den personenbezogenen Summenwerten a posteriori einer von drei Gruppen zugewiesen: gering lärmempfindlich (Summenwerte: $\leq 43 ; M=38,06, S D=4,87, n=51$ ), moderat lärmempfindlich (Summenwerte: $44-51 ; M=48,17, S D=$ $2,15, n=53$ ) und stark lärmempfindlich (Summenwerte: $\geq 52 ; M=57,24, S D=4,55, n=45$ ). Diese Gruppen unterscheiden sich hinsichtlich der individuellen Lärmempfindlichkeit signifikant voneinander, wie anhand des nichtparametrischen Kruskal-Wallis Tests bei nicht identischen Verteilungen festgestellt werden konnte, $H(2)=$ $131,56, p<, 001$.

Um gruppenbasierte Unterschiede von ordinalskalierten Variablen feststellen zu können, wurden ebenfalls mehrere nichtparametrische Kruskal-Wallis Tests durchgeführt. Die Lärmempfindlichkeit beeinflusst demnach die Häufigkeit der mobilen Musiknutzung, $H(2)=15,00, p=, 001$ (siehe Abbildung 1). Die anschließenden Post-hoc-Tests zwischen der gruppierten Lärmempfindlichkeit (Bonferroni-Korrektur) weisen darauf hin, dass Personen mit einer starken Lärmempfindlichkeit seltener unterwegs Musik hören als Personen mit einer geringen $(z=3,83, p<, 001, r=, 4)$ oder moderaten Lärmempfindlichkeit $(z=2,57, p=, 03, r=, 25)$.

Die Funktion „Ich höre Musik, um störende Geräusche auszublenden“ erhielt insgesamt unter den mobilen Musikhörer/-innen eher Zustimmung $(M=3,52, S D=1,29)$. Dass mobile Musikhörer/-innen unterwegs Musik nutzen, um störende Geräusche auszublenden, steht ebenfalls unter dem Einfluss der Lärmempfindlichkeit, wie ein weiterer Kruskal-Wallis-Test zeigte, $H(2)=10,79, p=, 005$ (siehe Abbildung 2). Die Post-hoc-Tests verdeutlichen, dass Personen mit einer geringen Lärmempfindlichkeit aus diesem Grund seltener unterwegs Musik hören als Personen mit einer starken $(z=-2,76, p=, 018, r=-, 30)$ oder moderaten Lärmempfindlichkeit $(z=-2,88, p=, 012, r=-, 29)$. 


\section{Abbildung 1}

Häufigkeit der mobilen Musiknutzung von Befragten mit geringer, moderater und starker Lärmempfindlichkeit

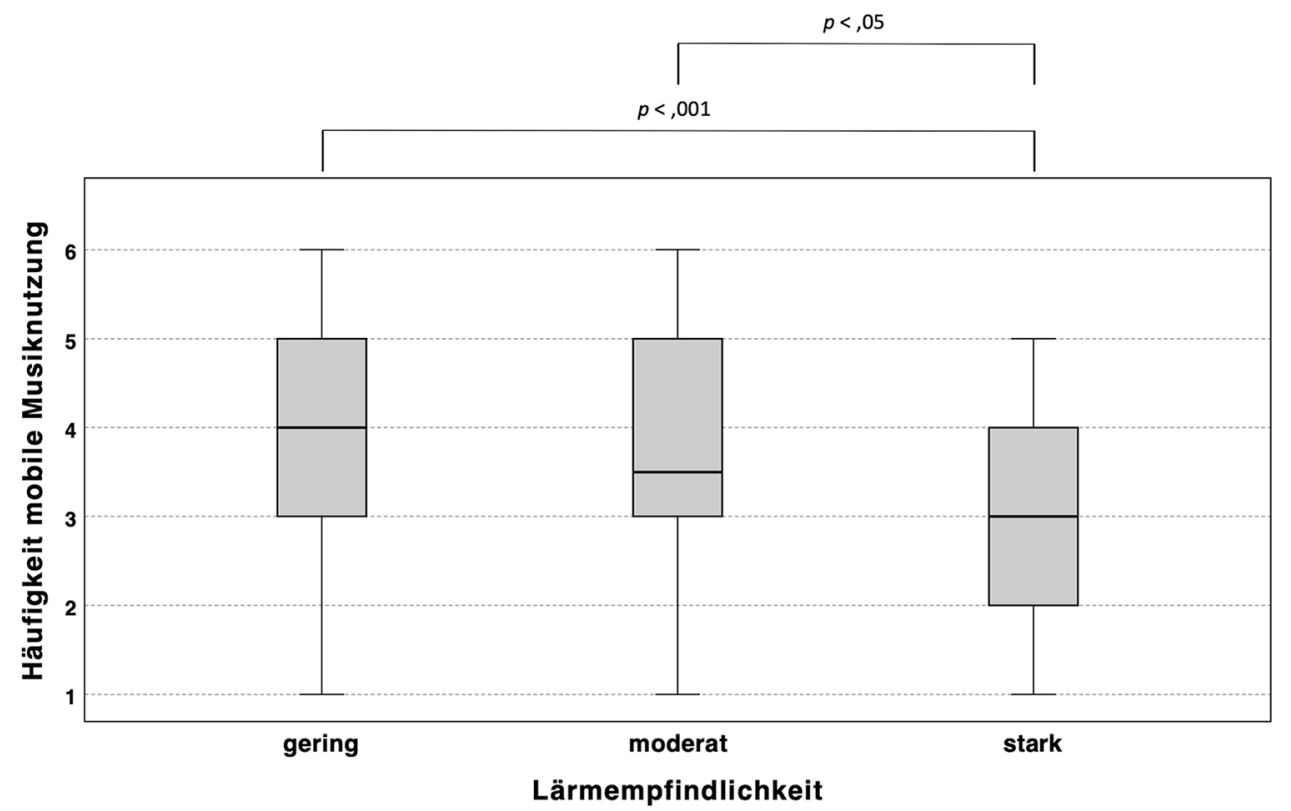

Anmerkung. Dargestellt sind die Gruppenunterschiede in der mobilen Musiknutzung (1 = nie bis $6=$ täglich 1-2 Stunden) nach Kruskal-Wallis und signifikanten Post-hoc-Tests. Boxplots mit Median, Interquartilsbereich, Minimum und Maximum.

\section{Abbildung 2}

Zustimmung zum mobilen Musiknutzungsgrund „Ausblenden störender Geräusche“ von Befragten mit geringer, moderater und starker Lärmempfindlichkeit

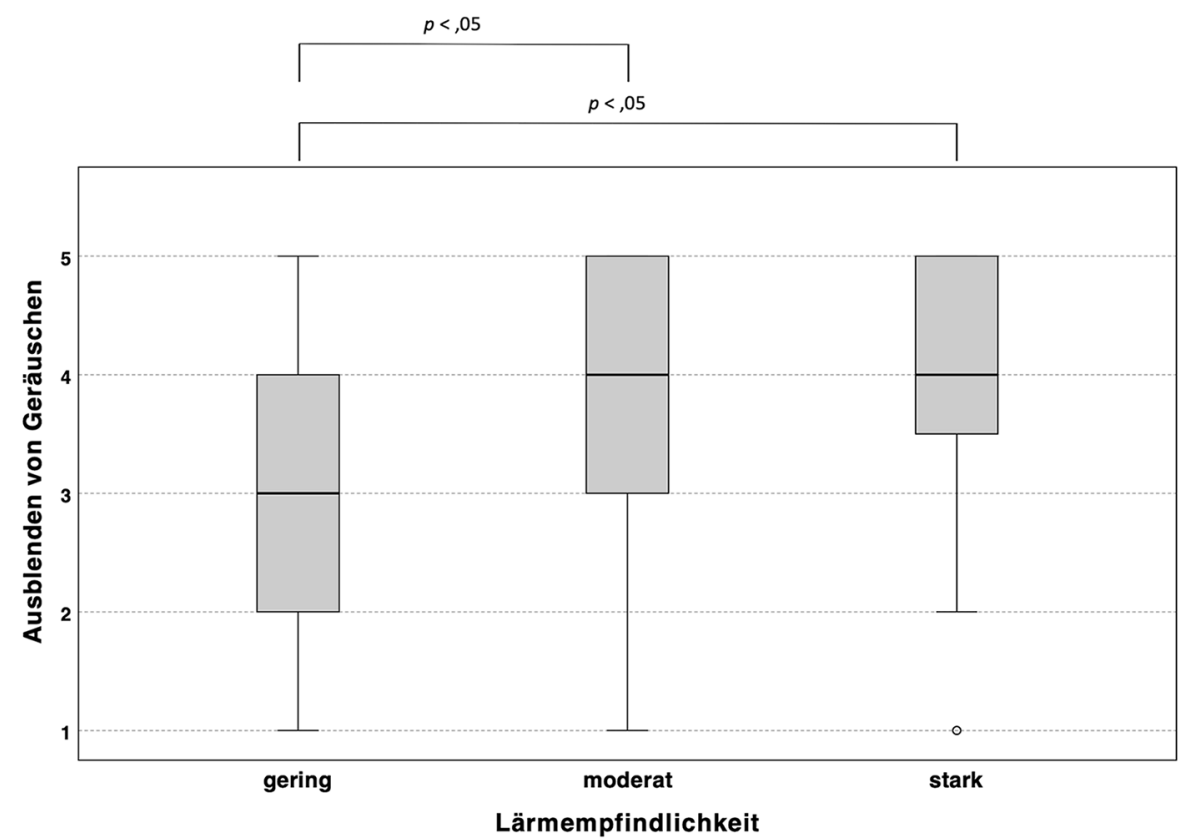

Anmerkung. Dargestellt sind die Gruppenunterschiede hinsichtlich der Zustimmung $(1=$ stimme überhaupt nicht zu bis $5=$ stimme voll und ganz zu) nach Kruskal-Wallis und signifikanten Post-hoc Tests. 


\section{Mobiles Musikhören und subjektive Lautheit in Abhängigkeit von den Verkehrsmitteln}

Am häufigsten waren die Befragten zu Fuß unterwegs $(M=2,64, S D=0,69)$, gefolgt von der $S$ - oder U-Bahn $(M=1,89, S D=1,12)$, während Motorrad oder Roller kaum genutzt wurden $(M=0,08, S D=0,36)$. Die verschiedenen Verkehrsmittel wurden einer von zwei entwickelten Kategorien zugeordnet, die sich hinsichtlich der individuellen Fortbewegung (zu Fuß, Auto, Fahrrad, Motorrad/Roller) oder der Nutzung von öffentlichen Verkehrsmitteln (S- oder U-Bahn, Bus, Regionalbahn, Straßenbahn) unterscheiden. Für beide Kategorien wurden individuelle Summenwerte nach der Häufigkeit der Nutzung berechnet und daraus anschließend ein individueller Differenzwert zur Art der Fortbewegung ermittelt. Basierend auf den Differenzwerten wurden drei Gruppen gebildet, wonach 37 Personen $(24,8 \%)$ überwiegend öffentliche Verkehrsmittel nutzen $(M=-3,49$, $S D=1,52)$, 53 Personen (35,6\%) ausgeglichen sowohl öffentliche Verkehrsmittel als auch individuelle Fortbewegungsarten nutzen $(M=0,19, S D=0,74)$, und schließlich 59 Personen $(39,6 \%)$ sich überwiegend individuell im urbanen Raum fortbewegen $(M=3,59, S D=1,48)$.

Der mögliche Einfluss der Verkehrsmittel auf das Hörverhalten wurde ebenfalls anhand eines Kruskal-Wallis-Tests untersucht. Dabei zeigte sich kein Unterschied hinsichtlich der Häufigkeit der mobilen Musiknutzung zwischen den Personen, die eher öffentliche, nicht-öffentliche Verkehrsmittel oder diese ausgeglichen nutzen, $H(2)=2,05, p=, 360$. Auch zeigte sich hier kein Unterschied hinsichtlich der Art der Verkehrsmittelnutzung und der subjektiven Einschätzung der Lautheit, mit der unterwegs mobil Musik gehört wird, $H(2)=0,38, p=, 826$.

\section{Zusammenhänge zwischen der Lärmempfindlichkeit mit der Stadtgröße, dem Alter, der allgemeinen Musiknutzung und den Hörgewohnheiten}

Ein Einfluss der Stadtgröße, in der sich die Befragten hauptsächlich aufhielten (von 5.000 bis mehr als 500.000 Einwohner), wurde anhand von Spearman-Korrelationen mit Bonferroni-Korrektur untersucht. Dabei zeigte sich weder ein signifikanter Zusammenhang mit der individuellen Lärmempfindlichkeit, $r_{\mathrm{s}}(147)=-, 17, p=, 074$, noch mit der subjektiv empfundenen Lautheit, mit der unterwegs Musik gehört wird, $r_{\mathrm{s}}(133)=, 16, p=, 152$.

Die individuelle Lärmempfindlichkeit steigt jedoch mit zunehmendem Alter an, $r_{\mathrm{s}}(149)=, 21, p=, 027$. Außerdem deuten die Ergebnisse darauf hin, dass mit zunehmender Lärmempfindlichkeit generell auch weniger aktiv Musik gehört wird, $r_{\mathrm{s}}(149)=-, 25, p=, 009$, und die unterwegs gehörte Musik der subjektiven Einschätzung nach leiser ist, $r_{\mathrm{s}}(135)=-, 25, p=, 012$ (Bonferroni-korrigiert).

Unter den mobilen Musikhörer/-innen wurden am häufigsten „In-Ear“-Kopfhörer (69,1\%) verwendet, weiterhin gaben 15,4\% an, „Over-Ear“-Kopfhörer, 14\% „On-Ear“-Kopfhörer und 9,6\%, „Earbuds“ oder „Earpods“ zu verwenden. Kopfhörer mit aktiver Geräuschunterdrückung (Noise-Cancelling) verwendeten nur 8,1\% der mobilen Musikhörer/-innen, während 9,6\% keine Kopfhörer verwendeten (Mehrfachnennungen möglich). Um die Kopfhörerwahl im Zusammenhang mit der Lärmempfindlichkeit untersuchen zu können, wurde eine weitere Variable generiert, welche die Nutzung von abschottenden Kopfhörern (Noise-Cancelling, Over-Ear, In-Ear) und nicht abschottenden Kopfhörern abbildet. Insgesamt verwendeten 107 Personen (78,7\% der mobil Musikhörenden) derartige Kopfhörer. Ein Chi-Quadrat-Test zeigte jedoch keinen Einfluss der gruppierten Lärmempfindlichkeit auf die Kopfhörerwahl, $X^{2}(2)=1,84, p=, 399$. 


\section{Diskussion}

Obwohl Lärm im Gesundheitsbereich schon seit Jahrzenten thematisiert wird und es einige Hinweise darauf gibt, dass Musikhören die empfundene Lärmbelastung reduzieren kann, findet Musikhören als Bewältigungsstrategie in der Forschung bis heute wenig Berücksichtigung. Bestehende Studien zur mobilen Musiknutzung im öffentlichen Raum bieten jedoch erste Hinweise darauf, dass Musik während des Unterwegsseins verwendet werden kann, um Umweltlärm auszublenden und das Belästigungsempfinden zu reduzieren (Bull, 2005; Heye \& Lamont, 2010; Skånland, 2011). Inwieweit sich Personen jedoch von Umweltlärm belästigt fühlen, ist individuell verschieden. Daher stellte sich die Frage, wie sich die individuelle Lärmempfindlichkeit auf die mobile Musiknutzung auswirkt.

Die vorliegende Untersuchung zeigt, dass lärmempfindliche Personen seltener unterwegs Musik hören als ihre weniger lärmempfindlichen Mitmenschen. Lärmempfindliche Personen bewerten Geräusche generell als lauter und unangenehmer (Ellermeier et al., 2001), und obwohl sich Musik in ihren Eigenschaften klar von Umweltgeräuschen unterscheidet (Reybrouck et al., 2019), könnte auch sie insbesondere von lärmempfindlichen Personen potenziell als belästigend wahrgenommen werden. Dies wird weiterhin von dem aktuellen Ergebnis gestützt, dass lärmempfindliche Personen auch generell weniger aktiv Musik hören als lärmunempfindlichere Personen. Daher kann wohl eine als belästigend empfundene Geräuschquelle (Umweltlärm) kaum durch eine andere Geräuschquelle (Musik) übertönt werden. Dennoch ist der Wunsch bei lärmempfindlichen Personen, sich von störenden Geräuschen abschotten zu wollen, besonders stark ausgeprägt, wenn sie unterwegs Musik hören. Möglicherweise verzichten lärmempfindliche Personen auf Musik, wenn sie können, greifen jedoch auf diese zurück, wenn der Lärm als zu störend empfunden wird. Gleichzeitig bewerten lärmempfindliche Personen die unterwegs gehörte Musik subjektiv als leiser. Um Umweltgeräusche ausblenden zu können, muss die Musik in der Lautstärke an die der Umweltgeräusche angepasst sein, woraus sich ableiten ließe, dass lärmempfindliche Personen nur bei geringer Lärmbelastung überhaupt Musik hören. Überschreiten die Umweltgeräusche eine gewisse Lautstärke, auf die mit einer Erhöhung der Musiklautstärke reagiert werden müsste, könnte dann die Musik selbst als belastend empfunden werden.

Für lärmunempfindliche Personen hingegen scheint die Funktion, störende Umweltgeräusche auszublenden, weniger entscheidend zu sein. Andere Nutzungsmotive, beispielsweise zur Affektregulation oder zum Zeitvertreib (siehe z. B. Bull, 2000; Kuch \& Wöllner, 2021), spielen womöglich eine größere Rolle als das akustische Maskieren, dem keine bewusste Intention zugrunde liegt (siehe auch Heye \& Lamont, 2010).

Die Ergebnisse liefern außerdem weitere Hinweise, die ein besseres Verständnis der Lärmempfindlichkeit im Zusammenhang mit der Musiknutzung ermöglichen. Mit zunehmendem Alter steigt auch in unserer Stichprobe die Lärmempfindlichkeit, was Erkenntnisse aus vorherigen Studien bestätigt (z. B. Shepherd et al., 2015; Miedema \& Vos, 1999). Die Größe der Stadt hingegen, in der sich die Befragten hauptsächlich aufhielten, zeigte weder einen Zusammenhang mit der Lärmempfindlichkeit noch mit der subjektiv empfundenen Lautheit der gehörten Musik. Dies könnte zum einen darauf zurückgeführt werden, dass nicht nur urbane, sondern auch ländliche Räume von Umweltgeräuschen betroffen sind, und ein Rückschluss auf die Lärmbelastung allein über die Stadtgröße nicht möglich ist. Zum anderen gaben jedoch knapp 60\% der Befragten an, sich im Alltag hauptsächlich in einer Großstadt ab 500.000 Einwohner/-innen aufzuhalten, wodurch nicht festzustellende Effekte auch in der geringen Datenvarianz begründet liegen könnten. 
Die mobile Musiknutzung unterschied sich nicht zwischen der Nutzung öffentlicher Verkehrsmittel und individuellen Fortbewegungsarten. Da auch die subjektiv empfundene Lautheit der Musik in Abhängigkeit der Verkehrsmittelwahl nicht variierte, ist davon auszugehen, dass sich individuell fortbewegende Personen (z. B. zu Fuß, mit dem Fahrrad) von hoher Lärmbelastung ebenso betroffen sein dürften wie diejenigen, die öffentliche Verkehrsmittel nutzen.

Eine weitere Bewältigungsstrategie von Lärm stellt potenziell die Nutzung von NC-Kopfhörern, Over-Ear- oder In-Ear-Kopfhörern dar, die auch ohne Musik eine effektive Abschottung gegen Lärm bieten können. Die Ergebnisse weisen jedoch nicht auf Zusammenhänge zwischen erhöhter Lärmempfindlichkeit und dem Tragen von abschottenden Kopfhörern hin (NC, Over-Ears, In-Ears).

\section{Einschränkungen und Ausblick}

Mit einem Umfang von 149 teilnehmenden Personen ist die Stichprobe relativ klein und umfasst zu einem großen Teil Studierende (46,1\%). Demographische und sozioökonomische Einflussvariablen auf die Lärmempfindlichkeit und Nutzung von Musik als Bewältigungsstrategie könnten womöglich weiterführende Erkenntnisse bieten. Daher wäre eine größere und heterogenere Stichprobe für nachfolgende Studien in diesem Feld wünschenswert.

Eine weitere Schwierigkeit stellt die Einschätzung der subjektiven Lärmempfindlichkeit dar, die neben anderen Faktoren entscheidend dafür ist, ab welchen Schallpegeln sich Personen von Geräuschen belästigt fühlen. Die Ergebnisse unserer Studie zeigen zwar eine individuell unterschiedlich stark ausgeprägte Lärmempfindlichkeit, jedoch nicht das Verhältnis der subjektiven Lärmempfindlichkeit zu objektiv messbaren Schallpegeln. Eine umfassende Messung der Geräuschpegel innerhalb einzelner Verkehrsmittel im deutschsprachigen Raum steht noch aus (siehe aber BMVI, 2019), wodurch erst ein Bezug zu den Empfehlungen der WHO hergestellt werden könnte. Eine detaillierte Untersuchung zu Lärmpegeln und individueller Lärmempfindlichkeit könnte nicht zuletzt darüber neue Erkenntnisse liefern, ob und inwiefern der Anteil der von Lärm betroffenen Bevölkerung steigt.

Darüber hinaus basieren die Einschätzungen der Lärmempfindlichkeit auf subjektiven Einstellungen zu einzelnen Aussagen. Die Entwicklung eines möglichst kurzen Fragebogens zur Messung der Lärmempfindlichkeit, der speziell auf den Kontext des Unterwegsseins und der mobilen Musiknutzung abzielt und dabei auch die individuelle Lärmbelastung berücksichtigt, wäre empfehlenswert. Mittels eines standardisierten Fragebogens könnte darüber hinaus die Einstufung des Lärmempfindlichkeitsgrads vereinheitlicht werden und müsste nicht in Abhängigkeit der Datenverteilung einer vorliegenden Stichprobe definiert werden. Um den Effekt der mobilen Musiknutzung weiterhin besser einschätzen zu können, erscheint eine Erhebung individueller Stressreaktionen ebenfalls vielversprechend. Der vorliegende Fragebogen kann als Ausgangspunkt dafür betrachtet werden, da sich durch vergleichbare Befunde in anderen Studien, wie dem Zusammenhang zwischen Lärmempfindlichkeit und Alter oder Häufigkeit der Musiknutzung, positive Rückschlüsse hinsichtlich der Auswahl der Items ziehen lassen.

Weiterhin sollten Befragungen direkt innerhalb öffentlicher Verkehrsmittel oder mittels „Experience Sampling Method“ (z. B. Sloboda et al., 2001; Randall \& Rickard, 2013) zuverlässigere Ergebnisse erzielen als retrospektive Befragungen, die potenziell durch Erinnerungsfehler (Recall Bias) verzerrt sein könnten. Vergleichbar mit der Studie von Heye und Lamont (2010) kann eine quantitative Vorgehensweise durch qualitative Methoden 
ergänzt werden, wodurch Einblicke in individuelle Bewältigungsstrategien ermöglicht werden. Auch ließen sich dadurch äußere Faktoren aufzeigen, die eine Lärmempfindlichkeit begünstigen.

Zusammenfassend zeigt die vorliegende Studie, dass sich lärmempfindliche Personen in ihrem Musiknutzungsverhalten im öffentlich-urbanen Raum von weniger lärmempfindlichen Personen unterscheiden. Die Ergebnisse bestätigen, dass mobiles Musikhören als Bewältigungsstrategie zur Lärmreduktion genutzt wird, wobei allerdings eine differenzierte Betrachtungsweise notwendig erscheint in Bezug auf die individuelle Lärmempfindlichkeit und die situativen Rahmenbedingungen im Kontext des mobilen Musikhörens. Die als relativ hoch eingeschätzte Lärmempfindlichkeit in den vorliegenden Daten weist zudem darauf hin, dass weitere Bemühungen zur Reduzierung von Lärm im öffentlichen Raum dringend geboten sind.

\section{Finanzierung}

Die Autoren/die Autorinnen haben keine Finanzierung für das Forschungsprojekt erhalten.

\section{Interessenkonflikte}

Die Autoren/Autorinnen haben erklärt, dass keinerlei konkurrierende Interessen bestehen.

\section{Danksagung}

Die Autoren/die Autorinnen haben keine weitere (d.h. nicht-finanzielle) Unterstützung erhalten.

\section{Literatur}

Airo, E., Pekkarinen, J., \& Olkinuora, P. (1996). Listening to music with earphones: An assessment of noise exposure. Acta Acustica united with Acustica, 82(6), 885-894.

Bull, M. (2000). Sounding out the city: Personal stereos and the management of everyday life. Berg.

Bull, M. (2005). No dead air! The iPod and the culture of mobile listening. Leisure Studies, 24(4), 343-355. https://doi.org/10.1080/0261436052000330447

Bundesministerium für Umwelt, Naturschutz und nukleare Sicherheit. (2014). Was ist Lärm? Abgerufen am 24. Juni 2021 von https://www.bmu.de/themen/luft-laerm-verkehr/laermschutz/laermschutz-im-ueberblick/was-ist-laerm/

Bundesministerium für Umwelt, Naturschutz und nukleare Sicherheit. (2017). Lärmwirkung. Abgerufen am 24. Juni 2021 von https://www.bmu.de/themen/luft-laerm-verkehr/laermschutz/laermschutz-im-ueberblick/laermwirkung/

Bundesministerium für Verkehr und digitale Infrastruktur. (2019). Lärmschutz im Schienenverkehr. Alles über Schallpegel, innovative Technik und Lärmschutz an der Quelle. Abgerufen am 01. Februar 2021 von https://www.bmvi.de /SharedDocs/DE/Publikationen/E/laermschutz-im-schienenverkehr-broschuere.pdf?_blob=publicationFile

Chen, S.-L. S. (1998). Electronic narcissism: College students' experiences of walkman listening. Qualitative Sociology, 21(3), 255-276. https://doi.org/10.1023/A:1022142519564

Ellermeier, W., Eigenstetter, M., \& Zimmer, K. (2001). Psychoacoustic correlates of individual noise sensitivity. The Journal of the Acoustical Society of America, 109(4), 1464-1473. https://doi.org/10.1121/1.1350402 
European Environment Agency. (2020). Environmental noise in Europe - 2020. Abgerufen am 25. Mai 2020 von https://www.courthousenews.com/wp-content/uploads/2020/03/Environment-noise-in-europe-2020_TH-AL-20-003-ENN.pdf

Haines, M. M., Brentnall, S. L., Stansfeld, S. A., \& Klineberg, E. (2003). Qualitative responses of children to environmental noise. Noise \& health, 5(19), 19-30. Abgerufen am 04. Juni 2021 von https://www.noiseandhealth.org/text.asp? 2003/5/19/19/31701

Heye, A., \& Lamont, A. (2010). Mobile listening situations in everyday life: The use of MP3 players while travelling. Musicae Scientiae, 14(1), 95-120. https://doi.org/10.1177/102986491001400104

Hosokawa, S. (1984). The walkman effect. Popular Music, 4, 165-180. https://doi.org/10.1017/S0261143000006218

Job, R. F. S. (1988). Community response to noise: A review of factors influencing the relationship between noise exposure and reaction. The Journal of the Acoustical Society of America, 83(3), 991-1001. https://doi.org/10.1121/1.396524

Kliuchko, M., Heinonen-Guzejev, M., Monacis, L., Gold, B. P., Heikkilä, K. V., Spinosa, V., Tervaniemi, M., \& Brattico, E. (2015). The association of noise sensitivity with music listening, training, and aptitude. Noise \& Health, 17(78), 350-357. https://doi.org/10.4103/1463-1741.165065

Koslowsky, M., Kluger, A. N., \& Reich, M. (1995). Commuting stress: Causes, effects, and methods of coping. Plenum Press.

Koushki, P. A., Ali, M. A., Chandrasekhar, B. P., \& Al-Sarawi, M. (2002). Exposure to noise inside transit buses in Kuwait: Measurements and passenger attitudes. Transport Reviews, 22(3), 295-308. https://doi.org/10.1080/01441640110103914

Kuch, M., \& Wöllner, C. (2021). On the move: Principal components of the functions and experiences of mobile music listening. Musicae Scientiae, 4, 1-14. https://doi.org/10.1177/20592043211032852

Lercher, P. (1996). Environmental noise and health: An integrated research perspective. Environment International, 22(1), 117-129. https://doi.org/10.1016/0160-4120(95)00109-3

Lundquist, P., Holmberg, K., \& Landstrom, U. (2000). Annoyance and effects on work from environmental noise at school. Noise \& health, 2(8), 39-46. Abgerufen am 04. Juni 2021 von https://www.noiseandhealth.org/text.asp?2000/2/8/39/31753

Miedema, H. M. E., \& Vos, H. (1999). Demographic and attitudinal factors that modify annoyance from transportation noise. The Journal of the Acoustical Society of America, 105, 3336-3344. https://doi.org/10.1121/1.424662

Miedema, H. M. E., \& Vos, H. (2003). Noise sensitivity and reactions to noise and other environmental conditions. The Journal of the Acoustical Society of America, 113(3), 1492-1504. https://doi.org/10.1121/1.1547437

Miyakawa, M., Matsui, T., Kishikawa, H., Murayama, R., Uchiyama, I., Itoh, T., \& Yoshida, T. (2006). Salivary chromogranin A as a measure of stress response to noise. Noise \& Health, 8(32), 108-113. https://doi.org/10.4103/1463-1741.33951

Neitzel, R., Gershon, R. R. M., Zeltser, M., Canton, A., \& Akram, M. (2009). Noise levels associated with New York City's mass transit systems. American Journal of Public Health, 99(8), 1393-1399. https://doi.org/10.2105/AJPH.2008.138297 
Ragettli, M. S., Goudreau, S., Plante, C., Perron, S., Fournier, M., \& Smargiassi, A. (2015). Annoyance from road traffic, trains, airplanes and from total environmental noise levels. International Journal of Environmental Research and Public Health, 13(1). https://doi.org/10.3390/ijerph13010090

Randall, W. M., \& Rickard, N. S. (2013). Development and trial of a mobile experience sampling method (m-ESM) for personal music listening. Music Perception, 31(2), 157-170. https://doi.org/10.1525/mp.2013.31.2.157

Randall, W. M., \& Rickard, N. S. (2017). Personal music listening: A model of emotional outcomes developed through mobile experience sampling. Music Perception, 34(5), 501-514. https://doi.org/10.1525/mp.2017.34.5.501

Reybrouck, M., Podlipniak, P., \& Welch, D. (2019). Music and noise: Same or different? What our body tells us. Frontiers in Psychology, 10, Article 1153. https://doi.org/10.3389/fpsyg.2019.01153

Rice, C. G., Breslin, M., \& Roper, R. G. (1987). Sound levels from personal cassette players. British Journal of Audiology, 21(4), 273-278. https://doi.org/10.3109/03005368709076419

Schutte, M., Marks, A., Wenning, E., \& Griefahn, B. (2007). The development of the noise sensitivity questionnaire. Noise \& Health, 9(34), 15-24. https://doi.org/10.4103/1463-1741.34700

Shepherd, D., Heinonen-Guzejev, M., Hautus, M. J., \& Heikkilä, K. (2015). Elucidating the relationship between noise sensitivity and personality. Noise \& Health, 17(76), 165-171. https://doi.org/10.4103/1463-1741.155850

Shimokura, R., \& Soeta, Y. (2012). Listening level of music through headphones in train car noise environments. The Journal of the Acoustical Society of America, 132(3), 1407-1416. https://doi.org/10.1121/1.4740472

Shu, H., Song, Y., \& Zhou, H. (2018). Assessment of music and water sounds for urban noise masking. In Proceedings of TENCON 2018 (Jeju, Korea, 28-31 October 2018). Abgerufen am 24. Juni 2021 von https://ieeexplore.ieee.org /stamp/stamp.jsp?tp=\&arnumber=8650456

Simun, M. (2009). My music, my world: Using the MP3 player to shape experience in London. New Media \& Society, 11(6), 921-941. https://doi.org/10.1177/1461444809336512

Skånland, M. S. (2011). Use of MP3 players as a coping resource. Music and Arts in Action, 3(2), 15-33.

Skånland, M. S. (2013). Everyday music listening and affect reg- ulation: The role of MP3 players. International Journal of Qualitative Studies on Health and Well-being, 8(1), Article 20595. https://doi.org/10.3402/qhw.v8i0.20595

Sloboda, J. A., O'Neill, S. A., \& Ivaldi, A. (2001). Functions of music in everyday life: An exploratory study using the experience sampling method. Musicae Scientiae, 5(1), 9-32. https://doi.org/10.1177/102986490100500102

Sung, J. H., Lee, J., Jeong, K. S., Lee, S., Lee, C., Jo, M.-W., \& Sim, C. S. (2017). Influence of transportation noise and noise sensitivity on annoyance: A cross-sectional study in South Korea. International Journal of Environmental Research and Public Health, 14(3). https://doi.org/10.3390/ijerph14030322

Tabacchi, M., Pavón, I., Ausejo, M., Asensio, C., \& Recuero, M. (2011). Assessment of noise exposure during commuting in the Madrid subway. Journal of Occupational and Environmental Hygiene, 8(9), 533-539. https://doi.org/10.1080/15459624.2011.600237

Umweltbundesamt. (2020). Gesundheitliche Belastungen durch Umweltverschmutzung und Lärm - Ergebnisse der Umweltbewusstseinsstudien. Abgerufen am 27. Juni 2021 von https://www.umweltbundesamt.de/sites 
/default/files/medien/2378/dokumente/ubs-2018-factsheet-gesundheitliche_belastungen_laerm_barrierefrei.pdf

Weinstein, N. D. (1978). Individual differences in reactions to noise: A longitudinal study in a college dormitory. The Journal of Applied Psychology, 63(4), 458-466. https://doi.org/10.1037/0021-9010.63.4.458

Wöllner, C., \& Hammerschmidt, D. (2021). Tapping to hip-hop: Effects of cognitive load, arousal, and musical meter on time experiences. Attention, Perception \& Psychophysics, 83, 1552-1561. https://doi.org/10.3758/s13414-020-02227-4

World Health Organization. (1999). Guidelines for community noise. Abgerufen am 25. September 2019 von https://apps.who.int/iris/bitstream/handle/10665/66217/a68672.pdf?sequence=1\&isAllowed=y

World Health Organization. (2018). Environmental noise guidelines for the European region. Abgerufen am 25. September 2019 von http://www.euro.who.int/_data/assets/pdf_file/0008/383921/noise-guidelines-eng.pdf?ua=1

Worthington, D. L. (2018). Weinstein noise sensitivity scale (WNSS). In D. L. Worthington \& D. B. Graham (Hrsg.), The sourcebook of listening research: methodology and measures (1. Aufl., S. 475-481). John Wileys \& Sons.

Yao, C. M. K. L., Ma, A. K., Cushing, S. L., \& Lin, V. Y. W. (2017). Noise exposure while commuting in Toronto - a study of personal and public transportation in Toronto. Journal of Otolaryngology - Head \& Neck Surgery, 46, Article 62. https://doi.org/10.1186/s40463-017-0239-6

Zimmer, K., \& Ellermeier, W. (1998). Konstruktion und Evaluation eines Fragebogens zur Erfassung der individuellen Lärmempfindlichkeit. Diagnostica, 44(1), 11-20.

\section{Anhang}

\section{Fragebogen}

\section{1) Musikhörverhalten}

Wie häufig hören Sie im Durchschnitt aktiv Musik?

(1) weniger als einmal in der Woche, (2) mehrmals in der Woche, (3) täglich unter einer Stunde, (4) täglich 1-2 Stunden, (5) täglich 2-4 Stunden, (6) täglich 4-8 Stunden, (7) täglich mehr als 8 Stunden

Bitte geben Sie an, welches Fortbewegungsmittel Sie wie häufig nutzen.

Auto, Straßenbahn, S- oder U-Bahn, Regionalbahn, Bus, Motorrad/Roller, Fahrrad, zu Fuß

(0) Nie, (1) selten, (2) gelegentlich, (3) oft

Wie häufig hören Sie im Durchschnitt unterwegs aktiv Musik?

(0) das kann ich nicht einschätzen, (1) ich höre unterwegs keine Musik, (2) mehrmals im Monat, (3) mehrmals in der Woche, (4) täglich unter einer Stunde, (5) täglich 1-2 Stunden, (6) täglich 2-4 Stunden, (7) täglich mehr als 4 Stunden

Wenn Sie unterwegs Musik hören, welche Art von Kopfhörern verwenden Sie? (Mehrfachantworten möglich)

NC (Noise-Cancelling), In-Ear, On-Ear (nicht Ohr-umschließend), Over-Ear (Ohr-umschließend), Earbuds/Earpods, ich nutze unterwegs keine Kopfhörer

Subjektiv eingeschätzt, wie laut ist die Musik, die Sie unterwegs hören meistens? 
(1) sehr leise, (2) leise, (3) eher leise, (4) mittlere Lautstärke, (5) eher laut, (6) laut, (7) sehr laut

\section{2) Nutzungsgrund des mobilen Musikhörens}

Bitte schätzen Sie die folgenden Aussagen ein. Sie beziehen sich auf das mobile Musikhören.

Ich höre Musik, um störende Geräusche auszublenden.

(1) Stimme überhaupt nicht zu, (2) stimme nicht zu, (3) weder noch, (4) stimme zu, (5) stimme voll und ganz zu

\section{3) Skala zur Messung der Lärmempfindlichkeit}

Bitte schätzen Sie die folgenden Aussagen ein. Sie beziehen sich auf die Wahrnehmung von Lärm.

1. Laute Unterhaltungen anderer stören mich nicht. $(\mathrm{N})$

2. Ich sehne mich häufig nach Stille.

3. Wenn es laut ist, bleibe ich meistens ruhig und entspannt. $(\mathrm{N})$

4. Lärmschutz wird zu wichtig genommen. (N)

5. Laute Menschen machen mich nervös.

6. Dem täglichen Straßenlärm versuche ich meistens möglichst schnell zu entkommen.

7. Ich bemerke störende Lärmquellen später als andere. $(\mathrm{N})$

8. Ich bin der Ansicht, dass während der Nachtzeit absolute Ruhe herrschen sollte.

9. Wenn es unterwegs laut ist, möchte ich etwas dagegen unternehmen.

10. Ich entspanne mich bei lauter Musik. (N)

11. Lärm stresst mich.

12. Ich bin geräuschempfindlich.

(1) Stimme überhaupt nicht zu, (2) stimme nicht zu, (3) stimme eher nicht zu, (4) stimme eher zu, (5) stimme zu,

(6) stimme voll und ganz zu

Anmerkung. (N) markiert negativ gepolte Items. Item 2, 4, 6, 7, 8, 9 und 10 wurden aus dem Fragebogen von Zimmer und Ellermeier (1998) entnommen, Item 12 aus dem Fragebogen von Worthington (2018; Weinstein, 1978). Item 1, 3 und 11 wurden für die vorliegende Studie neu entwickelt.

\section{4) Personenbezogene Daten}

Geschlecht

Weiblich, männlich, inter/divers, ich möchte dazu keine Angabe machen

Alter in Jahren

Wo halten Sie sich in Ihrem Alltag am häufigsten auf? 
(1) Landgemeinde unter 5000 Einwohner, (2) Kleinstadt 5.000 - 10.000 Einwohner, (3) Kleinstadt 10.000 - 20.000 Einwohner, (4) Mittelstadt 20.000 - 50.000 Einwohner, (5) Mittelstadt 50.000 - 100.000 Einwohner, (6) Großstadt 100.000 - 500.000 Einwohner, (7) Großstadt ab 500.000 Einwohner 